\title{
EL PAPEL DE LA AMPLIFICACIÓN SOMATOSENSORIAL EN LA PREDICCIÓN DE SÍNTOMAS RESPIRATORIOS
}

\author{
José CARMona ${ }^{1}$, Luis Miguel PASCUAL ${ }^{1}$, José Luis SÁNCHEZ ${ }^{2}$, José ANTONiO \\ MALDONADO $^{3}$ y ANTONIO PEREIRA ${ }^{3}$
}

\author{
${ }^{1}$ Departamento de Psicología, Universidad de Huelva \\ ${ }^{2}$ Departamento de Enfermería, Universidad de Huelva \\ ${ }^{3}$ Servicio de Neumología, Hospital Juan Ramón Jiménez, Huelva
}

\begin{abstract}
Resumen: La amplificación somatosensorial (ASS) es la tendencia a experimentar sensaciones somáticas como intensas, nocivas y perturbadoras (Barsky, 1992). Además de estar involucrada en los trastornos hipocondríacos, también podría explicar las diferencias en síntomas físicos que muestran pacientes con las mismas patologías. Estudios previos han comprobado que la ASS es el mejor predictor de algunos síntomas respiratorios, frente a otras variables como la ansiedad, la depresión o algunos indicadores objetivos (Muramatsu et al., 2002). Nuestro objetivo es examinar el papel de la ASS en la manifestación de síntomas respiratorios en población no clínica. Se han empleado técnicas de regresión logística jerárquica para determinar la capacidad predictiva de la ASS, controlando el efecto de sexo, edad, consumo de tabaco, ansiedad y preocupación por la salud. La variable que mejor predice la manifestación de síntomas es el consumo de tabaco. La ASS sólo muestra cierta capacidad predictiva en el caso de la disnea.
\end{abstract}

Palabras clave: Amplificación somatosensorial, síntomas respiratorios, percepción de síntomas

\section{The role of somatosensory amplification in the prediction of respiratory symptoms}

\begin{abstract}
Somatosensory amplification (ASS) is the tendency to experience somatic sensations as intense, harmful and disruptive (Barsky, 1992). Besides being involved in hypochondria disorders, it could explain the differences in physical symptoms between patients with the same pathologies. Previous studies have found that ASS is the best predictor of some respiratory symptoms, as opposed to other variables such as anxiety, depression or several objective indicators (Muramatsu et al., 2002). Our goal is to examine the role of somatosensory amplification in the manifestation of respiratory symptoms, in a non-clinical population sample. Hierarchical logistic regression models were used to determine the predictive capacity of ASS, after adjustment for sex, age, tobacco smoking, anxiety and health concern. Our findings show that smoking is the variable that best predicts the appearance of symptoms. ASS only shows limited predictive capacity as to dyspnoea.
\end{abstract}

Keywords: Somatosensory amplification, respiratory symptoms, symptoms perception.

\section{INTRODUCCIÓN}

El interés por el estudio de la amplificación somatosensorial (ASS) proviene de las investi-

Recibido 15 noviembre 2005; aceptado 21 diciembre 2006.

Correspondencia: Luis Miguel Pascual Orts, Departamento de Psicología, Universidad de Huelva. Avda Fuerzas Armadas 3, Campus del Carmen, 21071 Huelva. Correo-e: 1pascual@uhu.es.

Este trabajo se ha realizado mediante un proyecto de investigación financiado por la Comunidad Europea SI2.3032381 (2000 CVG2-609). gaciones realizadas sobre la hipocondría, donde se planteó inicialmente, desde una perspectiva cognitiva, que este trastorno podría ser producto de alteraciones derivadas del estilo perceptivo y/o atencional (Barsky, 1979; Barsky, Goodson, Lane y Cleary, 1988; Barsky y Klerman, 1983; García-Campayo, Pascual, Alda, y Oliván, 2005). El término «amplificación somatosensorial» fue propuesto por Barsky y colaboradores (Barsky, 1992; Barsky y Wyshak, 1990) para referirse a la tendencia de experimentar sensaciones somáticas como intensas, 
nocivas y perturbadoras. Esta tendencia incluiría la disposición individual para centrarse en sensaciones psicológicas y fisiológicas desagradables y considerarlas como patológicas antes que como normales.

Según estos autores, en la ASS se diferencian tres elementos: 1) Hipervigilancia corporal, que conlleva un aumento del autoescrutinio y del enfoque de la atención en sensaciones corporales desagradables; 2) tendencia a seleccionar y centrarse en ciertas sensaciones relativamente infrecuentes y/o tenues; 3 ) propensión a valorar las sensaciones somáticas y viscerales como anómalas, patológicas e indicadoras de enfermedad en vez de como normales. En definitiva, esta valoración cognitiva otorgaría una característica amenazadora y de alarma a la experiencia consciente de los síntomas (Muramatsu et al., 2002).

Desde estos planteamientos, la ASS desempeñaría un papel patogénico en la hipocondría y se relaciona de forma importante con otros indicadores de este trastorno como la conciencia corporal privada (Belloch, Ayllon, Martínez, Castañeiras y Jiménez, 1999) o las valoraciones cognitivas de amenaza (Ferguson et al., 2000). Se considera un indicador fiable de actitudes hipocondríacas en población general, aunque existen otros autores que plantean que es una variable poco sensible y específica para su utilización en el diagnóstico de la hipocondría (Speckens, Spinhoven, Sloekers, Bolk y Hemert, 1996; Speckens, Hemert, Spinhoven y Bolk, 1996). Esta variable constituiría también un síntoma inespecífico de otros trastornos mentales que incluyen síntomas físicos, como por ejemplo en el trastorno de pánico donde la ASS sería considerada como una reacción secundaria (Martínez, Belloch y Botella, 1999).

Por último, la ASS se hallaría presente en los procesos transitorios de somatización, no necesariamente patológicos (Barsky, 1992), y daría explicación de las diferencias en síntomas físicos que muestran pacientes con las mismas enfermedades, como por ejemplo en dolor crónico (Ak, Sayar y Yontem, 2004) o en trastornos isquémicos (Torosian, Lumley, Pickard y Ketterer, 1997). Teniendo en cuenta la presencia de esta variable en distintos estados, podríamos entenderla como una dimensión psicoló- gica continua, presente en todas las personas, donde los pacientes hipocondríacos ocuparían los lugares más extremos del continuo (Belloch et al., 1999).

Una cuestión a plantear es si aquellas personas, sin trastornos mentales, con una mayor tendencia a la focalización en sus signos corporales presentan también un mayor número de síntomas como consecuencia del escrutinio, selección y valoración anómala de dichos signos. Como plantean Barsky y Wyshak (1990), una mayor puntuación en ASS está asociada a la manifestación de síntomas de forma estable y duradera a lo largo del tiempo. Los resultados de estos estudios sugieren que la tendencia a amplificar está relacionada con la variabilidad individual en los pacientes con síntomas subjetivos y desagradables, incluso después de que las diferencias en la gravedad objetiva de la enfermedad de los pacientes hayan sido controladas estadísticamente.

La ASS converge con medidas autoinformadas como manifestación de síntomas, valoraciones médicas del malestar físico del paciente, preocupación corporal o preocupación por la enfermedad, pero no existen las mismas relaciones cuando se contrasta con medidas objetivas de sensibilidad corporal como la evaluación de la frecuencia de los latidos del corazón (Barsky, Brener, Coeytaux, y Cleary, 1995; Barsky, Cleary, Brener y Ruskin, 1993; Mailloux y Brener, 1998; 2002). Más específicamente relacionado con este trabajo, distintos estudios han encontrado relaciones significativas entre los autoinformes de amplificación y quejas somáticas en pacientes con infecciones del tracto respiratorio superior (Barsky et al., 1988; Muramatsu et al., 2002). Según datos propios preliminares (Morales et al., 2004), las personas con síntomas respiratorios tenían una mayor puntuación en ASS, aunque solamente se encontraron diferencias estadísticamente significativas en síntomas como disnea, tos y esputos.

Ante estas evidencias, es importante determinar cual es el papel que está jugando la ASS en la manifestación de síntomas. Algunos estudios anteriores han comprobado que la ASS es el mejor predictor de los síntomas respiratorios examinados, frente a otras variables como la ansiedad, la depresión o los indicadores objeti- 
vos (Muramatsu et al., 2002). En este trabajo, para determinar el valor predictivo de la ASS hemos tomado en cuenta diferentes variables (tabaquismo, ansiedad, preocupación por la salud, sexo y edad) que pueden influir en la manifestación de síntomas (Fabbri, Kapur, Wells y Reed, 2001) y hemos elaborado un modelo estadístico para contrastar el efecto de la ASS controlando dichas variables.

Nuestro objetivo en este estudio es examinar el papel que desempeña la amplificación somatosensorial en la manifestación de síntomas respiratorios, en una muestra de población no clínica.

\section{MÉTODO}

\section{Participantes}

La muestra inicial de este estudio constaba de 707 personas y se obtuvo a partir del seguimiento realizado a los participantes en dos estudios previos (Gómez et al., 1997; European Community Respiratory Health Survey —ECRHS—, 1996): (a) Jóvenes de 18-23 años participantes en el estudio epidemiológico de asma en adolescentes de la ciudad de Huelva (FIS 99/0350); representan a los adolescentes que en 1993 estaban escolarizados en $6 .^{\circ}, 7 .^{\circ} \mathrm{y}$ 8. ${ }^{\circ}$ de EGB en la ciudad de Huelva; y (b) adultos de 30-54 años participantes en el estudio europeo de incidencia del asma y factores de riesgo (ECRHS) (FIS 99/0034-06); representan el seguimiento de la cohorte seleccionada al azar de la población de Huelva de 20 a 44 años realizado en 1991.

Los criterios de inclusión en nuestro estudio fueron: adecuada comprensión lectora, no padecer enfermedades respiratorias crónicas (asma o bronquitis) y responder de manera adecuada a los cuestionarios psicológicos. De este modo, la muestra final constaba de 319 participantes ( $45,1 \%$ de la muestra inicial) y estaba compuesta por 170 hombres y 149 mujeres. La edad media de los participantes en el estudio era de 28,18 años $(D T=11,48)$. Sólo un $35,1 \%$ de los participantes fumaba actualmente. El resto se distribuía entre un $52,4 \%$ que nunca había fumado y un $12,5 \%$ de ex-fumadores. Se comprobó que no existían diferencias significativas $(p<0,01)$ entre estos valores y los correspondientes en la muestra completa.

\section{Instrumentos}

Cuestionario ECRHS (Burney, Luczynska, Chinn y Jarvis, 1994): incluye preguntas sobre síntomas respiratorios y hábito tabáquico. Para el presente estudio fueron seleccionados los siguientes síntomas respiratorios: sibilancias (¿Ha tenido silbidos o pitos en el pecho alguna vez en los últimos 12 meses?), tos (¿Tose habitualmente al levantarse por la mañana durante el invierno?; ¿Tose habitualmente de día o de noche durante el invierno?), esputos (¿Acostumbra a arrancar o sacar esputos al levantarse por la mañana durante el invierno?; ¿Acostumbra a arrancar o sacar esputos durante el día o la noche en invierno?) y disnea ( $ఓ H a$ tenido algún ataque de falta de aire durante el día, estando en reposo?; ¿Ha tenido algún ataque de falta de aire después de hacer ejercicio físico en los últimos doce meses?). Una respuesta afirmativa a cualquiera de los ítems indicados era considerada como indicador del síntoma correspondiente.

Escala de amplificación somatosensorial (Barsky, Wyshak y Klerman, 1990): Consiste en 10 items que incluyen sensaciones corporales benignas que son molestas pero que no constituyen síntomas típicos de enfermedad. Estos ítems son estimados en una escala de cinco puntos (desde Nada a Muchísimo). El rango posible de puntuaciones oscila entre 10 y 55 . Se utilizó la versión española adaptada por Martínez et al. (1999).

Inventario de ansiedad rasgo (STAI-R: Spielberger, Gorsuch y Lushene, 1982): comprende una escala de autoevaluación que mide ansiedad. Cada escala contiene 20 ítems con cuatro alternativas de respuesta «Casi nunca, A veces, A menudo y Casi siempre», con una puntuación de 0 a 3 según su carga y dirección hacia la ansiedad. Una puntuación de 38 se considera como indicadora de trastornos de ansiedad. Los elementos del STAI son suficien- 
temente discriminantes y diferenciadores (en variables como la edad o el sexo) y poseen una buena consistencia interna (entre 0,84 y 0,87 ) según los análisis realizados para la adaptación española (Seisdedos, 1988).

Escala de preocupación hacia la salud (Castañeiras, 2000): 16 preguntas relativas al nivel de preocupación y de rumiaciones en torno a la salud que se contestan en una escala Likert de 5 puntos (desde «No es en absoluto típico en mi» hasta «Es muy típico en mi»). El rango posible de puntuaciones oscila entre 16 y 80 .

\section{Procedimiento}

Los participantes en los estudios ECHRS y Estudio Epidemiológico sobre incidencia del asma en adolescentes de la ciudad de Huelva fueron citados para su seguimiento en el Hospital Juan Ramón Jiménez de Huelva. Las citas fueron realizadas originalmente por teléfono. Cuando no era posible la localización telefónica de los participantes, se enviaba una carta a su dirección postal. Una vez en el Hospital, los participantes fueron sometidos a varias pruebas por personal de enfermería específicamente entrenado, que incluían entre otras el cuestionario de síntomas respiratorios ECHRS. Además se les entregaban los cuestionarios psicológicos, junto con un sobre franqueado, para que los rellenaran en su domicilio y los enviaran posteriormente al Hospital. Después de un mes de la visita al Hospital, a las personas que no los habían enviado se les remitía una carta recordatorio con otra copia de los cuestionarios. Tras este segundo envío se obtuvo una tasa definitiva de respuesta a los cuestionarios psicológicos del 52,3\%. Todos los participantes fueron debidamente informados de la finalidad del estudio y dieron su consentimiento voluntario para participar en el mismo.

\section{Análisis estadísticos}

Para determinar la capacidad predictiva de la ASS se han empleado técnicas de regresión logística jerárquica y por pasos. Se usan técni- cas de regresión logística porque los criterios a predecir son variables dicotómicas (los participantes presentan o no presentan un determinado síntoma). Se usa una estrategia jerárquica para determinar si la ASS permite predecir la manifestación de síntomas, una vez controlado el efecto de otras variables predictoras.

En esta estrategia jerárquica se introduce en un primer paso un bloque de variables de control: el sexo, la edad, el consumo de tabaco (fumador/no fumador), la ansiedad-rasgo (STAI-R) y la preocupación por la salud (EPS). Estas variables predictoras constituyen el modelo predictivo base con el que contrastar la capacidad predictiva de la ASS. Para una mayor claridad en la interpretación de los resultados, en el análisis de regresión logística las puntuaciones en las escalas psicológicas fueron las medias, en lugar de las sumas, de las puntuaciones en cada ítem.

A continuación se introduce la ASS. El modelo final incluye por tanto las variables de control más la ASS. La evaluación del ajuste del modelo a los datos se realiza mediante una prueba de significación ( $\chi^{2}$ del modelo) y un estadístico del tamaño del efecto: $\mathrm{R}^{2}$ de Nagelkerke. La evaluación de la capacidad predictiva de la ASS, se realizará comparando el modelo final con el modelo base. La significación de la diferencia entre las $\chi^{2}$ de los dos modelos y la diferencia entre valores de la $\mathrm{R}^{2}$ de Nagelkerke serán los indicadores de esa capacidad predictiva.

\section{RESULTADOS}

Para evitar elementos de confusión en la manifestación de síntomas respiratorios, se han eliminado de la muestra total los sujetos que tenían enfermedades respiratorias crónicas (asma o bronquitis) por lo que el número total de la muestra general es de 602 sujetos. Aunque los porcentajes en las variables analizadas (tabaquismo, género, edad, tos, sibilancias, esputos o disnea) son algo inferiores en nuestra muestra de estudio (Figura 1), en ningún caso existen diferencias significativas $(p<0,01)$ con respecto a la muestra inicial por lo que consi- 


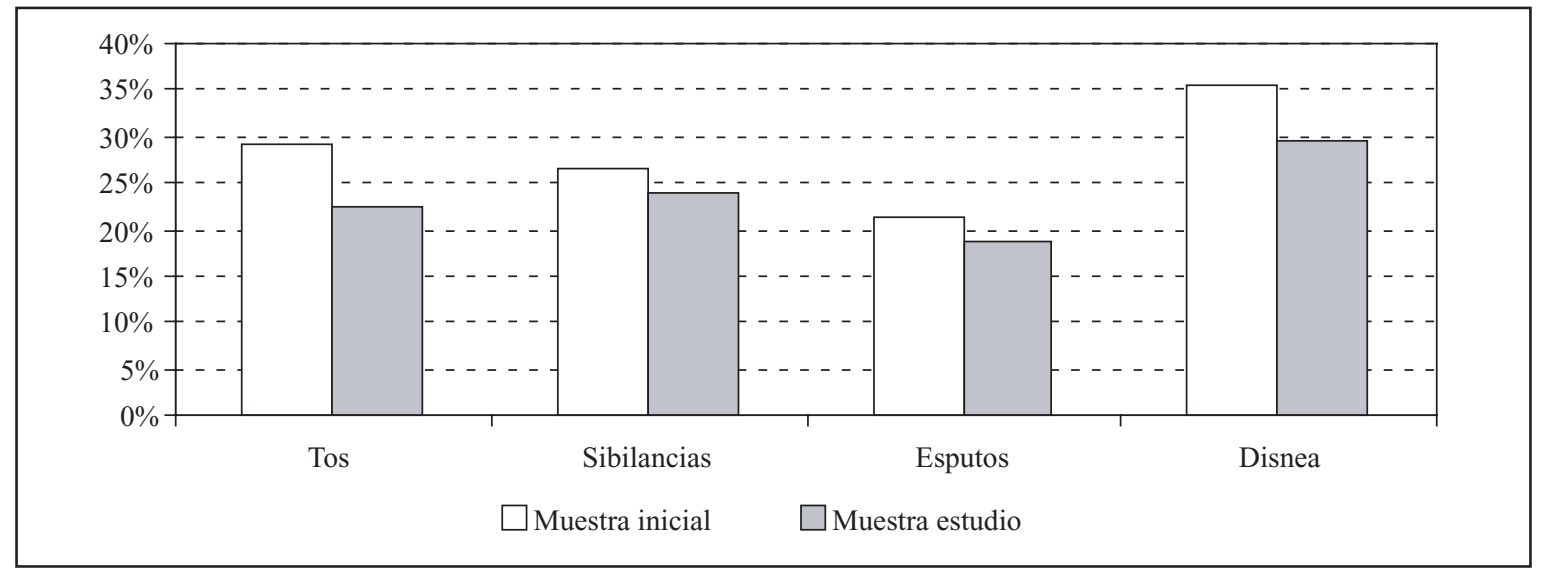

Figura 1: Comparación de la prevalencia de la sintomatología en la muestra inicial y la muestra de estudio

deramos generalizables los resultados obtenidos a la población general.

La fiabilidad de las escalas psicológicas entendida como consistencia interna a través del índice alfa de Cronbach obtuvo valores aceptables en la escala de la $\operatorname{ASS}\left(\chi^{2}=0,72\right)$ y buenos en el inventario de ansiedad rasgo $\left(\chi^{2}=\right.$ $0,87)$ y en la escala de preocupación por la salud $\left(\chi^{2}=0,85\right)$. La ASS obtuvo relaciones moderadas tanto con la ansiedad rasgo $(r=$ $0,39 ; p<0,01)$, como con la escala de preocupación por la salud $(r=0,37 ; p<0,01)$. La mujeres muestran una ASS media significativamente mayor que los hombres $(M=27,05$; $D T=6,23 ; \mathrm{M}=24,99 ; D T=6,07 ; \mathrm{t}=2,98$; $p=0,003)$. En cambio, no se encontraron relaciones significativas entre la edad de los participantes y la ASS $(r=-0,04 ; p=0,43)$.

Los resultados de los análisis de regresión logística muestran que la variable con una mayor capacidad predictiva de la manifestación de síntomas es el consumo de tabaco. Los fumadores tienen una probabilidad muy superior de manifestar síntomas que los no fumadores, con odds ratios superiores a cinco para la predicción de la tos, las sibilancias y los esputos (Tabla 1). Las otras variables que componían el modelo base (sexo, edad, preocupación por la salud y ansiedad rasgo), sin embargo, no resultan predictores significativos de ninguno de los síntomas analizados. Los valores de $R^{2}$ del modelo base muestran una capacidad predictiva moderada, salvo en el caso de la disnea, en la que ninguna de las variables de este modelo base se convierte en un predictor significativo.

La ASS sólo muestra cierta capacidad predictiva cuando el síntoma manifestado a predecir es la disnea. Mientras que el modelo base que incluye todas las demás variables tiene una $R^{2}$ igual a 0,05 , la inclusión de la ASS hace aumentar el valor de $R^{2}$ hasta 0,08 . Esta ganancia predictiva producida al incluir la ASS, aunque pequeña, es estadísticamente significativa a un nivel de confianza del $99 \%$. La odds ratio correspondiente $(O R=1,82)$ indica que el cociente entre la probabilidad de manifestar disnea y la probabilidad de no manifestar este síntoma (odds) se incrementa en un $82 \%$ por cada punto adicional en la escala de la ASS, una vez controlado el efecto de las otras variables predictoras incluidas en el modelo.

\section{DISCUSIÓN}

Desde los planteamientos del grupo de Barsky se planteaba que la ASS, además del papel patogénico que desempeña en la hipocondría, se hallaría presente en otras situaciones no necesariamente patológicas. De este modo, las personas con una disposición amplificadora manifestarían más síntomas que las personas no amplificadoras (Barsky, 1992). El objetivo de nuestro estudio ha sido comprobar el papel predictivo de la ASS en la manifestación de síntomas respiratorios en una muestra de población 
Tabla 1. Odds ratios de los síntomas respiratorios para las variables incluidas en el modelo final

\begin{tabular}{lcccc}
\hline & Tos & Sibilancias & Esputos & Disnea \\
\hline Variables de control & & & & \\
Sexo & $0,70(0,31-1,56)$ & $1,66(0,75-3,67)$ & $2,34(0,97-5,62)$ & $1,48(0,74-2,94)$ \\
Edad & $1,01(0,98-1,04)$ & $1,02(0,99-1,05)$ & $0,99(0,96-1,03)$ & $0,99(0,96-1,02)$ \\
Tabaco & $6,55^{*}(2,99-14,35)$ & $5,48^{*}(2,61-11,51)$ & $5,31^{*}(2,35-12,00)$ & $1,70(0,87-3,33)$ \\
EPS & $1,61(0,83-3,13)$ & $1,29(0,68-2,45)$ & $1,09(0,55-2,16)$ & $1,45(0,81-2,60)$ \\
STAI-R & $1,27(0,52-3,07)$ & $1,46(0,61-3,49)$ & $1,10(0,43-2,83)$ & $0,72(0,32-1,59)$ \\
\hline Variable de prueba & & & & \\
ASS & $1,45(0,72-2,92)$ & $1,09(0,55-2,14)$ & $1,87(0,90-3,90)$ & $1,82 *(1,00-3,30)$ \\
\hline Modelo base & $\chi^{2}(5)=52,97$ & $\chi^{2}(5)=44,31$ & $\chi^{2}(5)=35,55$ & $\chi^{2}(5)=11,72$ \\
& $p<0,01$ & $p<0,01$ & $p<0,01$ & $p=0,04$ \\
Modelo final & $R^{2}=0,23$ & $R^{2}=0,19$ & $R^{2}=0,17$ & $R^{2}=0,05$ \\
& $\chi^{2}(6)=54,80$ & $\chi^{2}(6)=44,42$ & $\chi^{2}(6)=40,55$ & $\chi^{2}(6)=18,60$ \\
& $p<0,01$ & $p<0,01$ & $p<0,01$ & $p<0,01$ \\
\hline Ganancia predictiva & $R^{2}=0,24$ & $R^{2}=0,19$ & $R^{2}=0,19$ & $R^{2}=0,08$ \\
& $\chi^{2}(1)=1,84$ & $\chi^{2}(1)=0,11$ & $\chi^{2}(1)=5,00$ & $\chi^{2}(1)=6,87$ \\
& $p=0,17$ & $p=0,75$ & $p=0,02$ & $p<0,01$ \\
& $R^{2}=0,01$ & $R^{2}=0,00$ & $R^{2}=0,02$ & $R^{2}=0,03$ \\
\hline
\end{tabular}

Nota. Entre paréntesis se encuentra el intervalo de confianza para las odds ratios $(p<0,001)$. * $p<0,01$.

no clínica, controlando el efecto de otras variables relacionadas (sexo, edad, tabaquismo, ansiedad, preocupación por la salud).

En primer lugar, comprobamos que los datos sobre fiabilidad de la escala de la ASS obtenidos en nuestra muestra $\left(\chi^{2}=0,72\right)$ son similares a los que muestran otros autores en muestras de población general no clínica. Speckens, Spinhoven et al. (1996) obtuvieron valores $\chi$ de 0,71 en una muestra de población general con una media de edad de 39,9 $(D T=12,6)$ años y Belloch et al. (1999), con una muestra más joven $(M=28,75 ; D T=11,98$ años) compuesta mayoritariamente por estudiantes $(63 \%)$, obtuvieron un $\chi^{2}$ de Cronbach de 0,80 .

Las relaciones de la ASS con otras variables son también similares a las que se ofrecen en estudios previos. Con respecto al sexo, se pone de manifiesto la tendencia de las mujeres a una mayor puntuación en ASS (Speckens, Spinhoven et al., 1996; Belloch et al., 1999), aunque nuestros resultados no muestran relaciones entre la edad y la ASS como en otros trabajos (Belloch et al., 1999). Las relaciones de la ASS que aportan otras investigaciones con variables psicológicas como la ansiedad, la depresión o la preocupación por la salud son significativas, pero con valores moderados por debajo de 0,5 (Speckens, van Hemert et al., 1996; Kosturek, Gregory, Sousou y Trief, 1998; Aronson, Barrett y Quigley, 2001; Nakao, Barsky, Kumano y Kuboki, 2002), del mismo modo que en nuestros datos.

En cuanto al papel predictor de la ASS en la manifestación de síntomas respiratorios, en nuestros resultados sólo se alcanza cierta capacidad predictiva en la disnea, pero no en los demás síntomas incluidos como tos, esputos o sibilancias. Hay que tener en cuenta que el modelo analizado incluía como variable de control el consumo de tabaco y es precisamente esta variable la que tiene un mayor valor predictivo. Si no hubiese sido incluida, o la muestra hubiese sido exclusivamente de personas no fumadoras, el efecto de la ASS hubiese sido probablemente mayor.

Los resultados obtenidos en trabajos previos donde se ha examinado el papel predictivo de la ASS para los síntomas no son concluyentes. En un estudio, con otro tipo de trastorno, donde se comparaba las características de enfermos con isquemia de miocardio sintomáticos y asintomáticos se encontró que la ASS no tenía capacidad predictiva a través de un análisis de 
regresión donde se controlaba el efecto de la edad, el sexo y la raza. Los mejores predictores del grupo sintomático fueron el control de la ira y el pensamiento orientado al exterior (Torosian et al., 1997). En cambio, en el estudio de Muramatsu et al. (2002), con síntomas respiratorios, la ASS era el mejor predictor, tanto del número total de síntomas respiratorios como del malestar general, explicando el $20 \%$ de la varianza. En el análisis de regresión se incluían como variables independientes la edad, el sexo, los indicadores objetivos, la ansiedad y la depresión.

Las diferencias con el estudio de Muramatsu et al. (2002) pueden deberse a tres cuestiones: en primer lugar, en su estudio no se incluyó el tabaquismo en los análisis realizados para evaluar el papel predictivo de la ASS. Como hemos observado anteriormente, es precisamente el tabaquismo la mejor variable predictora en todos los síntomas respiratorios. Al incluir esta variable en nuestro modelo, el efecto de la ASS en la predicción de los síntomas queda minimizado. En segundo lugar, el tipo de muestra empleada por estos autores proviene de enfermos con infecciones del tracto respiratorio superior, mientras que en nuestro caso hemos realizado el estudio con sujetos de la población general, no clínica. Excluimos intencionadamente las personas con algún tipo de trastorno respiratorio para que el efecto de la ASS no quedase enmascarado por la sintomatología debida a las enfermedades respiratorias. Por último, mientras que nosotros sólo evaluamos la presencia o no de determinados síntomas durante el año anterior, el indicador de la sintomatología empleado en su trabajo era una escala donde el paciente evaluaba, en una escala tipo Likert de 5 puntos, hasta qué punto cada síntoma (de un total de 11) le había molestado durante las últimas veinticuatro horas. De este modo, no se estaría examinando tanto la manifestación o no del síntoma en referencia a la ASS, sino el impacto que los síntomas presentes estaban produciendo en el paciente.

La disnea, que se caracteriza por su causalidad multifactorial, es el único síntoma en el que la ASS muestra cierta capacidad predictiva. Sin embargo, la ganancia predictiva que ofrece la inclusión de la ASS, aunque estadísticamente significativa, es pequeña. En los otros síntomas, las variables que componen el modelo base explican mucho más que en la disnea, donde el porcentaje de varianza explicado es sólo de un $5 \%$. En este caso, la ASS aporta un 3\% más al modelo inicial. Como exponen De Peutera et al. (2004), la percepción de la disnea está modulada por las emociones (especialmente por las emociones negativas), tanto si están directamente relacionadas con la condición que causa la disnea como si no. Esta mayor importancia de las variables psicológicas en su manifestación, con las que está asociada como comprobamos anteriormente, facilitaría el papel de la ASS en su predicción.

Además de las diferencias metodológicas, y teniendo en cuenta las relaciones sistemáticas de la ASS con otras variables psicológicas, podría preguntarse, como plantean Aronson et al. (2001), si la ASS es más un índice de emocionalidad negativa que de sensibilidad somática, por lo que, al controlar el efecto de dichas variables, la relación de la ASS con la manifestación de síntomas queda minimizada.

En definitiva, y a la vista de nuestros resultados, debemos confirmar que la ASS no tiene un papel relevante en la predicción de síntomas respiratorios en población no clínica. La capacidad predictiva de la ASS para los síntomas respiratorios analizados se limita a la disnea, y de manera pequeña. La ASS no se muestra un buen predictor de estos síntomas, en oposición a estudios previos en los que se confirmaba un buen papel predictivo de la ASS, pero no se tenía en cuenta el papel del tabaquismo.

\section{REFERENCIAS}

Ak, I., Sayar, K., y Yontem, T. (2004). Alexithymia, somatosensory amplification and counter-dependency in patients with chronic pain. The Pain Clinic, 16, 43-51.

Aronson, K.R., Barrett, L.F., y Quigley, K.S. (2001). Feeling your body or feeling badly. Evidence for the limited validity of the Somatosensory Amplification Scale as an index of somatic sensitivity. Journal of psychosomatic research, 51, 387-394.

Barsky, A.J. (1979). Patients who amplify bodily sensations. Annals of International Medicine, 91, 63-70.

Barsky, A.J. (1992). Amplification, somatization ande the somatoform disorders. Psychosomatics, 33, 28-34. 
Barsky, A.J., Brener, J., Coeytaux, J.J., y Cleary, P.D. (1995). Accurate awareness of resting heartbeat in hypochondriacal and non-hypochondriacal patients. Journal of Psychosomatic Research, 39, 489-497.

Barsky, A.J., Cleary, P.D., Brener, J., y Ruskin, J.N., (1993). The perception of cardiac activity in medical outpatients. Cardiology, 83, 304-315.

Barsky, A.J., Goodson, J.D., Lane, R.S., y Cleary, P.D. (1988). The amplification of somatic symptoms. Psychosomatic Medicine, 50, 510-519.

Barsky, A.J., y Klerman, GL (1983). Overview: hypochondriasis, bodily complaints, and somatic styles. American Journal of Psychiatry, 140, 273-283.

Barsky, A.J., y Wyshak, G. (1990). Hypocondriasis and somatosensory amplification. British Journal of Psychiatry, 157, 404-409.

Barsky, A.J., Wyshak, G., y Klerman, GL (1990). The somatosensory amplification scale and its relationship to hypochondriasis. Journal of Psychiatric Research, 24, 323-334

Belloch, A., Ayllon, E., Martínez, M.P., Castañeiras, C., y Jimenez, M.A. (1999). Sensibilidad a las sensaciones corporals e hipocondría. Revista de Psicopatología y Psicología Clínica, 4, 1-14.

Burney, P., Luczynska, C., Chinn, S., y Jarvis, D. (1994). The European Community Respiratory Health Survey. European Respiratory Journal, 7, 954-60.

Castañeiras, C. (2000). Avances en psicopatología y clinica de la hipocondría. Tesis doctoral no publicada, Universidad de Valencia, Valencia.

De Peutera, S., Van Diesta, I., Lemaigreb, V., Verledenb, G., Demedtsb, M., y Van den Bergh, O. (2004). Dyspnoea: the role of psychological processes. Clinical Psychological Review, 24, 557-581.

European Community Respiratory Health Survey (1996). Variations in the prevalence of respiratory symptoms, self-reported asthma attacks, and use of asthma medication in the European Community Respiratory Health Survey (ECRHS). European Respiratory Journal, 9 , 687-695

Fabbri, S., Kapur, N., Wells, A., y Reed, F. (2001). Emotional, cognitive, and behavioral characteristics of medical outpatients: a prelimiray study. Psychosomatics, 42 (1), 74-77.

Ferguson, E., Swairbrick, R., Clare, S., Ronbisnon, E., Bignell, C.J., y Anderson, C. (2000). Hypochondriacal concerns, somatosensory amplification and primary and secondary cognitive appraisals. British Journal of Medical Psychology, 73, 355-369.

García-Campayo, J., Pascual, A., Alda, M., y Oliván, B. (2005). Tratamiento psicológico en el trastorno de somatización: Eficacia y propuestas de intervención. Revista de Psicopatología y Psicología Clínica, 10, 15-23.
Gómez, M., Pereira, A., Maldonado, J.A., Sánchez, J.L., Grávalos, J., y Delgado, R. (1997). Síntomas respiratorios, metacolina y tests cutáneos en población escolar. Revista Neumosur, 9, 160-164.

Kosturek, A., Gregory, R.J., Sousou, A.J., y Trief, P. (1998). Alexithymia and somatic amplification in chronic pain. Psychosomatics, 39 (5), 399-404.

Mailloux, J., y Brener, J. (1998, Mayo). Somatosensory amplification does not reflect sensitivity to heartbeat sensations. Comunicación presentada al 10 congreso anual de la American Psychological Society, Washington, DC.

Mailloux, J., y Brener, J. (2002). Somatosensory Amplification and its Relationship to Heartbeat Detection Ability. Psychosomatic Medicine, 64, 353-357.

Martinez, M.P., Belloch, A., y Botella, C. (1999). Somatosensory Amplification in Hypochondriasis and Panic Disorder. Clinical Psychological Psychotherapy, 6, 46-53.

Morales, Z., Pascual, L.M., Carmona, J., Sánchez J.L., Maldonado, J.A., y Pereira, A. (2004, Marzo). Amplificación somatosensorial y manifestación de síntomas respiratorios en una muestra de población general. Comunicación presentada al IV Congreso de la Asociación Española de Psicología Clínica y Psicopatología, Sevilla.

Muramatsu, K., Miyaoka, H., Muramatsu, Y., Fuse, K., Yoshimine, F., Kamijima, K., Gejyo, F., y Sakurai, K. (2002). The amplification of somatic symptoms in upper respiratory tract infections. General Hospital Psychiatry, 24, 172-175.

Nakao, M., Barsky, A.J., Kumano, H., y Kuboki, T. (2002). Relationships between somatosensory amplification and alexithymia in a japanese psychosomatic clinic. Psychosomatics, 43, 50-60.

Seisdedos, N. (1988). Cuestionario de Ansiedad EstadoRasgo. Adaptación española. Madrid, T.E.A.

Speckens, A.E.M., Hemert, A.M., Spinhoven, P., y Bolk, J.H. (1996). The diagnostic and prognostic significance of the Whitely Index, the Illness Attittude Scales and the Somatosensory Amplification Scale. Psychological Medicine, 26, 1085-1090.

Speckens, A.E.M., Spinhoven, P., Sloekers, P.P.A., Bolk, J.H., y Hemert, A.M. (1996). A validation study of the Whitely Index, the Illness Attittude Scales and the Somatosensory Amplification Scale in general medical and general practice patients. Journal of Psychosomatic Research, 40, 95-104.

Spielberger, C.D., Gorsuch, R.L., y Lushene, R.E. (1982): Cuestionario de Ansiedad Estado-Rasgo. Madrid, TEA.

Torosian, T., Lumley, M.A., Pickard, S.D., y Ketterer, M.W., (1997). Silent versus symptomatic myocardial ischemia: the role of psychological and medical factors. Health Psychology, 16, 123-130. 